\title{
Structural Construction for On-Line Mathematical Formulae Recognition
}

\author{
Daniel Průša and Václav Hlaváč \\ Czech Technical University, Faculty of Electrical Engineering \\ Department for Cybernetics, Center for Machine Perception \\ 12135 Prague 2, Karlovo náměstí 13 \\ Fax: + 420224357 385; Phone: + 420224357465 \\ \{prusa, hlavac\}@cmp.felk.cvut.cz
}

\begin{abstract}
We present a method for on-line mathematical formulae recognition based on the structural construction paradigm and twodimensional grammars. In general, this approach can be successfully used in the analysis of inputs composed of objects that exhibit rich structural relations. An important benefit of the structural construction is in not treating symbols segmentation and structural analysis as two separate processes which allows the system to perform segmentation in the context of the whole formula structure - this helps to solve arising ambiguities more reliably. We show that the proposed method can be effectively implemented and practically used.
\end{abstract}

Keywords: formulae recognition, two-dimensional grammars.

\section{Introduction}

Mathematical formulae recognition is a task of growing importance. It can be applied to digitize mathematical texts in scanned images (off-line recognition) or to handle mathematical inputs written on tablets (on-line recognition).

In this paper, we propose a method for on-line formulae recognition. Our approach is based on so called structural construction. Its very general idea was presented in [8]. We use a kind of two-dimensional (2D) grammars to express spatial relations among elementary mathematical symbols. In general, 2D parsing could be a difficult task since time complexity can grow exponentially in the number of elementary symbols. This is reason why some authors introduced nongrammar based systems like virtual link networks [3] or formalisms like graph grammars [5] allowing to perform the structural analysis on the basis of local relations only. In our case, a 2D grammar better suits our needs for searching a formula structure that fits the input strokes the best, with the lowest overall penalty of the derivation. An additional advantage of this approach is that the parsing ensures we obtain a syntactically correct $2 \mathrm{D}$ structure, no additional verifications are needed as e.g. in 9 .

In systems for formulae recognition proposed by others, we can quite often find that the segmentation is done prior to the structural analysis. Segmentation 
errors are eventually corrected by some postprocessing. This is true in almost all methods referenced by a taxonomy in [2]. There are some recent works where local properties of mathematical structures are taken into account when performing the segmentation, e.g. in [10]. We go further and introduce a method where the resolution of ambiguities that arise during symbols recognition and segmentation is fully driven by the parsing algorithm. This is one of our main achievements. Figure 1 shows simple examples of segmentation ambiguities that can be resolved by the proposed method.

The process we introduce has two phases. The elementary symbols detection phase tries to find each group of strokes that can form an elementary symbol. It is done without any knowledge of the formula structure, but the chosen groups are just candidates for the symbols. The structural analysis phase takes these candidates as the input, decides which of them really represent a symbol in the written formula and determines mathematical relations among them.

In the past, we already developed a pilot study for the off-line formulae recognition and published related results in 6]. Comparing to that work, we present an adoption of the method for the on-line data where inputs are sequences of strokes rather than raster images. It requires to propose a completely different strategy for the detection of elementary symbols candidates and to modify the structural analysis, especially the used data structures. Furthermore, we present significant improvements in the parsing algorithm resulting in a fast and responsive implementation.

The ideas of structural construction [8] have been also applied by others using simplified 2D grammars to the off-line recognition of musical scores [7] and electrical circuits 4. This paper is the first application for the on-line recognition.

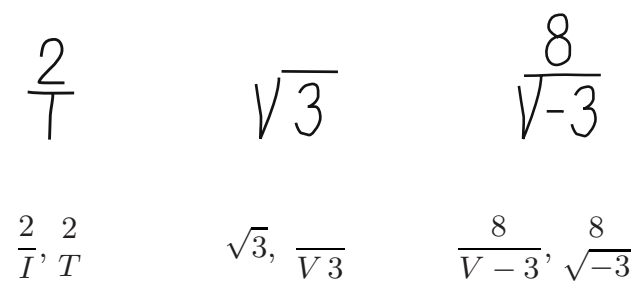

Fig. 1. Segmentation ambiguities. The pair under each hand-written formula describes an expected interpretation followed by an incorrect interpretation.

\section{Elementary Symbols Detection}

Definition 1. A stroke is a finite sequence $s=\left(\left(x_{1}, y_{1}\right), \ldots,\left(x_{k}, y_{k}\right)\right)$ where each $x_{i}, y_{i} \in \mathbb{R}$.

Informally, a stroke is a sequence of points in a plane. Strokes data are usually produced by tablets. Except coordinates, it is also possible to obtain information on a pen pressure and time of creation, however, we do not use this in our method. A sequence of strokes $S=\left(s_{1}, \ldots, s_{n}\right)$ forms an input to the recognition process. 
The purpose of the elementary symbols detection phase is to detect candidates for the formula elementary symbols. To suppress the large number of possibilities, we have implemented a strategy where only some groups of strokes are evaluated by an OCR tool for on-line character recognition. The strategy is based on the assumptions that one stroke cannot be a part of two or more different symbols and that a symbol is formed of at most 4 strokes. Moreover, we choose only groups of neighboring strokes. The criterions are formalized in the following paragraphs.

Definition 2. The distance between strokes

$$
\begin{aligned}
& s=\left(\left(x_{1}, y_{1}\right), \ldots,\left(x_{k}, y_{k}\right)\right) \\
& \bar{s}=\left(\left(\bar{x}_{1}, \bar{y}_{1}\right), \ldots,\left(\bar{x}_{l}, \bar{y}_{l}\right)\right)
\end{aligned}
$$

is defined as

$$
d(s, \bar{s})=\min _{i=1, \ldots, k ; j=1, \ldots, l} \sqrt{\left(x_{i}-x_{j}\right)^{2}+\left(y_{i}-y_{j}\right)^{2}}
$$

Let $S=\left(s_{1}, \ldots, s_{n}\right)$ be an input. We define a non-oriented graph $\mathbf{G}=(S, E)$ where

$$
\left\{s_{i}, s_{j}\right\} \in E \Longleftrightarrow i \neq j \wedge d\left(s_{i}, s_{j}\right)<c
$$

In the condition, we use a global constant $c$ determined empirically with respect to the training data set.

Let $\mathcal{C}_{1}(S)$ be a set containing groups of strokes where $S^{\prime} \subseteq S$ is in $\mathcal{C}_{1}(S)$ just when the following conditions are fulfilled:

$-1 \leq\left|S^{\prime}\right| \leq 4$

- Subgraph of $\mathbf{G}$ induced by nodes in $S^{\prime}$ is a connected graph.

Each $S^{\prime} \in \mathcal{C}_{1}(S)$ is evaluated by the OCR tool whether it can be recognized as an elementary symbol.

Definition 3. A labeled group of strokes over $S$ and a set of labels $V$ is a tuple $\left(S^{\prime}, l, p, M\right)$ where $S^{\prime} \subseteq S, l \in V, p \in \mathbb{R}^{+}$is a penalty, $M$ is a metrics record.

We use labeled groups of strokes to represent results returned by the OCR tool: $V$ is the set of all symbol names learned by the tool, $l$ corresponds the recognized symbol, $p$ expresses a reliability of the recognition (a lower value implies a bigger similarity to learned patterns) and $M$ is a record storing information on the symbol's base line, logical center and bounding box (its size and coordinates). We denote the set of all metrics records by $\mathfrak{M}$.

For each $S^{\prime}$, the tool returns up to 5 best matches. Furthermore, no result where the penalty exceeds a global threshold constant is returned. Finally, we can define a set of candidates $\mathcal{C}(S)$ containing all elements $\left(S^{\prime}, l_{i}, p_{i}, M_{i}\right)$ returned by the OCR tool when it runs for each $S^{\prime} \in \mathcal{C}_{1}(S)$. A candidates example is depicted in Figure 2. 


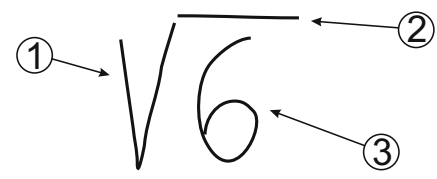

\begin{tabular}{c|c|c|c|c|c} 
symbol & variable $V$ & number 6 & fraction line & minus sign & square root \\
\hline strokes & 1 & 3 & 2 & 2 & 1,2
\end{tabular}

Fig. 2. A sequence of three strokes, found candidates are listed in the table

\section{Two-Dimensional Grammars}

Definition 4. A 2D co-ordinal grammar is a tuple $\mathcal{G}=\left(V_{T}, V_{N}, A_{0}, \mathcal{P}\right)$, where

- $V_{T}$ is a finite set of terminal symbols (terminals)

- $V_{N}$ is a finite set of non-terminal symbols (non-terminals)

- $A_{0} \in V_{N}$ is the starting non-terminal symbol (axiom)

$-\mathcal{P}$ is a finite set of $2 D$ productions

Given a set of candidates $\mathcal{C}(S)$ and a 2D co-ordinal grammar (modelling mathematical constructs in our case), the task of the structural analysis is to incrementally derive new labeled groups of strokes over $S$ and $V_{T} \cup V_{N}$. The result is chosen among those derived elements $\left(S^{\prime}, N, p, M\right)$ where $N=A_{0}$. Details on this will be given in Section 4. Informally, the goal is to minimize $p$ and also the number of points in $S \backslash S^{\prime}$.

We describe the form of productions in $\mathcal{P}$ and rules of how they are used to derive new elements. There are two types of productions:
1) $(N \rightarrow l)$
2) $(N \rightarrow A \oplus B, \sigma, \pi, \mu)$

where

- $N \in V_{N}, l \in V_{T}$ and $A, B \in V_{T} \cup\left(V_{N} \backslash\left\{A_{0}\right\}\right)$

$-\sigma: \mathfrak{M} \times \mathfrak{M} \rightarrow\{$ true, false $\}$ is a spatial constraint function

$-\pi: \mathfrak{M} \times \mathfrak{M} \rightarrow \mathbb{R}^{+}$is a penalty function

$-\mu: \mathfrak{M} \times \mathfrak{M} \rightarrow \mathfrak{M}$ is a metrics record composition function

Let

$$
R_{1}=\left(S_{1}, l_{1}, p_{1}, M_{1}\right) \quad R_{2}=\left(S_{2}, l_{2}, p_{2}, M_{2}\right)
$$

be labeled groups of strokes. A production of the first type is just a simple renaming. If there is $\left(N \rightarrow l_{1}\right) \in \mathcal{P}$, it is possible to derive $\left(S_{1}, N, p_{1}, M_{1}\right)$ from $R_{1}$. A production of the second type is used to derive a union of two elements: $(N \rightarrow A \oplus B, \sigma, \pi, \mu) \in \mathcal{P}$ can be applied on $R_{1}, R_{2}$ iff

$$
S_{1} \cap S_{2}=\emptyset \wedge l_{1}=A \wedge l_{2}=B \wedge \sigma\left(R_{1}, R_{2}\right)=\text { true }
$$

The following labeled group of strokes is derived

$$
R=\left(S_{1} \cup S_{2}, N, p_{1}+p_{2}+\pi\left(R_{1}, R_{2}\right), \mu\left(M_{1}, M_{2}\right)\right)
$$



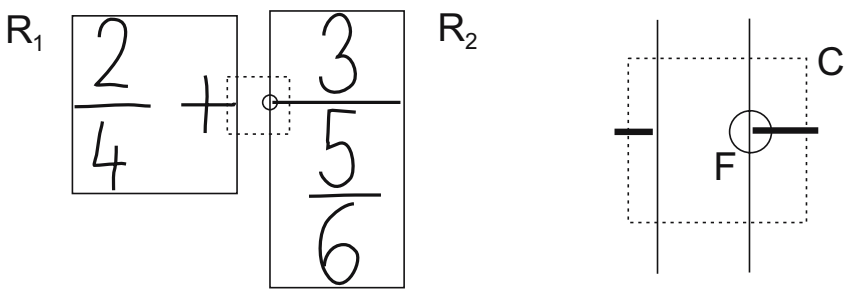

Fig. 3. A spatial constraint evaluation example. On the right, there is a zoomed reference point $F$ and constraining rectangle $C$. Related production is (BinaryOperation $\rightarrow$ ExpressionFollowedByBinaryOperator $\oplus$ Expression, $\sigma, \pi, \mu)$.

We give some details on $\sigma$ and $\pi$ we use in our implementation. Let $\operatorname{box}\left(S^{\prime}\right)$ denote bounding box of a sequence of strokes $S^{\prime}$. For $R_{1}$ and $R_{2}$, production's spatial constraint evaluates mutual position of $\operatorname{box}\left(S_{1}\right)$ and $\operatorname{box}\left(S_{2}\right)$. First, a rectangle $C$ is computed which of size and position is relative to box $\left(S_{1}\right)$. And second, it is checked whether some specific point of box $\left(S_{2}\right)$ (called a reference point), determined based on $M_{2}$, is contained in $C$. This is demonstrated in Figure 3 which shows an example of $R_{1}$ and $R_{2}$. The reference point, denoted by $F$, is required to be located in $C$.

A constraint can be quite general, we do not require the bounding boxes to touch each other, they can overlap or even more, one bounding box can be included in the other one, as it is used to model the spacial relations between square roots and their arguments.

$\pi\left(S_{1}, S_{2}\right)$ is computed based on the distance between $F$ and the center of $C$. Let $\left(f_{1}, f_{2}\right)$, resp. $\left(c_{1}, c_{2}\right)$ be coordinates of $F$, resp. the center of $C$. Then

$$
\pi\left(S_{1}, S_{2}\right)=w_{1} \cdot\left|f_{1}-c_{1}\right|+w_{2} \cdot\left|f_{2}-c_{2}\right|
$$

where $w_{1}, w_{2} \in \mathbb{R}$ are production dependent weight constants.

$\mu\left(M_{1}, M_{2}\right)$ computes a metrics record of the derived mathematical structure in terms of $M_{1}$ and $M_{2}$.

\section{Parsing Algorithm}

This section describes the parsing algorithm used by the structural analysis phase. Let $\mathcal{G}=\left(V_{T}, V_{N}, A_{0}, \mathcal{P}\right)$ be a $2 \mathrm{D}$ co-ordinal grammar and $S=\left(s_{1}, \ldots, s_{n}\right)$ an input sequence of strokes.

1. Initialize a list $\mathcal{L}$ by all labeled groups of strokes found by the terminals detection phase, i.e. append all elements in $\mathcal{C}(S)$ to $\mathcal{L}$.

2. For each $\left(S^{\prime}, l, p, M\right) \in \mathcal{L}$ and each $(N \rightarrow l) \in \mathcal{P}$, append $\left(S^{\prime}, N, p, M\right)$ to $\mathcal{L}$.

3. Iterate through elements in $\mathcal{L}$, repeat the following procedure till the end of $\mathcal{L}$ is reached.

Let $R_{1}=\left(S_{1}, l_{1}, p_{1}, M_{1}\right)$ be the current element in $\mathcal{L}$. For each production $(N \rightarrow A \oplus B, \sigma, \pi, \mu) \in \mathcal{P}$ where $l_{1}=A$, and each

$$
R_{2}=\left(S_{2}, l_{2}, p_{2}, M_{2}\right)
$$


such that

$$
\sigma\left(R_{1}, R_{2}\right)=\text { true } \wedge S_{1} \cap S_{2}=\emptyset
$$

Let $\bar{p}=p_{1}+p_{2}+\pi\left(S_{1}, S_{2}\right)$. Check if $\mathcal{L}$ contains an element

$$
R=\left(S_{1} \cup S_{2}, N, p, M\right)
$$

for some $p$ and $M$.

3.1 If $R$ is found and $\bar{p}<p$, append $\left(S_{1} \cup S_{2}, N, \bar{p}, \mu\left(M_{1}, M_{2}\right)\right)$ to $\mathcal{L}$ and remove $R$ from $\mathcal{L}$, otherwise do nothing.

3.2 If there is no $R$, append $\left(S_{1} \cup S_{2}, N, \bar{p}, \mu\left(M_{1}, M_{2}\right)\right)$ to $\mathcal{L}$.

Handle analogously the case $l_{1}=B$.

4. Compute a list $\mathcal{L}_{1}$ such that

$$
\mathcal{L}_{1}=\left\{\left(S^{\prime}, A_{0}, p+\sum_{s \in S \backslash S^{\prime}}|s|, M\right) \mid\left(S^{\prime}, A_{0}, p, M\right) \in \mathcal{L}\right\}
$$

Among elements in $\mathcal{L}_{1}$, find that one with the lowest penalty and return it as the result.

To have the description readable, we have omitted implementation specific details speeding-up the algorithm:

To search for $R_{2}$ in step 3 effectively, we use a data structure storing points in a plane, supporting to query for all points located inside a given rectangle. We speak about the orthogonal range searching [1]. A query is processed in time $\mathcal{O}(\log n)$, where $n$ is the number of stored points.

In the implementation, we have extended the condition (1) in step 3 to be more restrictive. In addition to (1), when the applied production does not model a relation where box $\left(S_{1}\right)$ can be located inside box $\left(S_{2}\right)$ (like e.g. a square root argument can), or vice versa, it is required that there is not any stroke $s \in$ $S \backslash\left\{S_{1} \cup S_{2}\right\}$ which of all points are located inside box $\left(S_{1} \cup S_{2}\right)$. This requirement substantially reduces the number of derived labeled groups of strokes, on the other hand, it still preserves the correctness of the structural analysis.

During the algorithm, whenever a new labeled group of strokes is appended to $\mathcal{L}$, information on the production and groups of strokes used to derive it is recorded. This helps to construct the resulting derivation tree.

\section{$5 \quad$ Experiments}

We have implemented the described recognition method in Java. Except the recognition, our implementation provides some additional functionality. For example, it allows to display detailed information on resulting derivation trees and also to enter and evaluate queries on these trees - the user can query for derived groups of strokes labeled by a specific non-terminal, penalties of related derivations, etc. This helps to understand and tune the process of the structural analysis. The used OCR tool, integrated in the elementary symbols detection 
phase, is a modified existing application, freely available at [11]. It implements the elastic matching technique. ACECAD DigiMemo notepad has been used to produce formulae. It persists on-line data in special .DHW files. We have adopted this proprietary file format to represent the training and testing formulae sets.

The designed 2D co-ordinal grammar allows to recognize usual mathematical symbols and constructs as numbers, variables, brackets, subscripts, superscripts, basic unary and binary operators, power to operations, fractions, sums, integrals and square roots.

The following table summarizes results of experiments we have carried out on a notebook equipped by Intel Core 2 Duo $2.00 \mathrm{GHz}$ processor.

\begin{tabular}{|c|c|c|c|c|c|c|c|}
\hline & $\begin{array}{c}\text { total } \\
\text { formulae }\end{array}$ & $\begin{array}{c}\text { total } \\
\text { errors }\end{array}$ & $\begin{array}{c}\text { OCR } \\
\text { errors }\end{array}$ & $\begin{array}{c}\text { correct } \\
\text { rate 1 }\end{array}$ & $\begin{array}{c}\text { correct } \\
\text { rate 2 }\end{array}$ & $\begin{array}{c}\text { total } \\
\text { time }\end{array}$ & $\begin{array}{c}\text { average time } \\
\text { per formula }\end{array}$ \\
\hline result & 440 & 49 & 36 & $88.8 \%$ & $97.0 \%$ & $36.1[\mathrm{~s}]$ & $0.082[\mathrm{~s}]$ \\
\hline
\end{tabular}

We give two correctness rates. Rate 1 is computed as the ratio between the total number of errors and the number of processed formulae. Rate 2 is computed after excluding the formulae unrecognized purely due to OCR failures. This correct rate better evaluates the quality of the proposed method. We have had an OCR tool of a moderate correctness rate only. An increase in rate 1 can be expected when a better tool is used instead. Figure 4 shows an example of recognized formula as well as examples of recognition failures. The computed average time spent to recognize a formula shows that the implemented parsing algorithm is really fast, making the recognition process responsive.

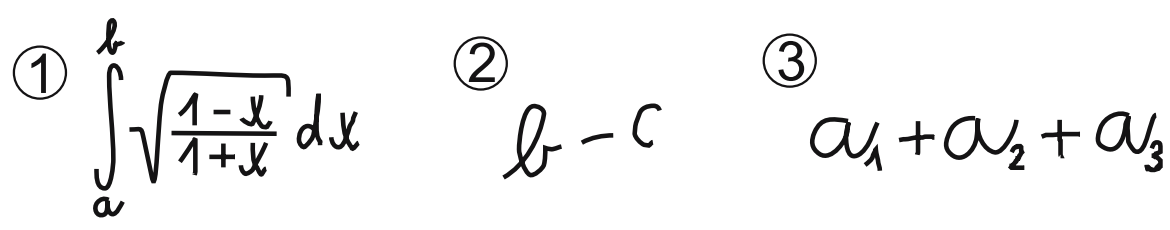

Fig. 4. The first formula has been successfully recognized, the second formula has not been parsed by the grammar, the third formula has been recognized as $a+a_{2}+a_{3}$ because of an OCR error (symbol 1 has not been recognized at all)

\section{Conclusion}

We have contributed to the problematic of the on-line mathematical formulae recognition by a method driving the strokes segmentation by structural analysis. The proposed analysis is robust. The strategy for elementary symbols detection differs to that one we presented for the off-line formulae recognition in [6]. It is more natural and elegant, since it is sufficient to evaluate groups of strokes. This is not true in the off-line case where we had to search trough rectangular areas of pixels. Many problems occurred with overlapping bounding boxes of elementary symbols. They had a relatively high impact on the correctness rate. 
In future, we would like to extend the designed 2D grammar to support more mathematical constructs, find possibilities of how to learn and tune constraints assigned to productions automatically (so far they have been set manually) and improve or replace the OCR tool to achieve a higher OCR correctness rate.

\section{Acknowledgement}

This work was supported by the Czech Ministry of Education - project 1M0567.

\section{References}

1. Berg, M., Schwarzkopf, O., Kreveld, M., Overmars, M.: Computational Geometry: Algorithms and Applications. Springer, Heidelberg (2000)

2. Chan, K.-F., Yeung, D.-Y.: Mathematical Expression Recognition: a Survey. International Journal on Document Analysis and Recognition 3, 3-15 (2000)

3. Eto, Y., Suzuki, M.: Mathematical Formula Recognition Using Virtual Network. In: Proceedings of ICDAR 2001, pp. 762-767. IEEE Computer Society, Los Alamitos (2001)

4. Kiyko, V.M.: Recognition of Objects in Images of Paper Based Line Drawings. In: Proceedings of ICDAR 1995, pp. 970-973. IEEE Computer Society, Washington (1995)

5. Lavirotte, S., Pottier, L.: Mathematical Formula Recognition Using Graph Grammar. In: Proceedings of SPIE 1998, San Jose, vol. 3305, pp. 44-52 (1998)

6. Prusa, D., Hlavac, V.: Mathematical Formulae Recognition using 2D Grammars. In: Proceedings of ICDAR 2007, Curitiba, vol. 2, pp. 849-853 (2007)

7. Savchynsky, B., Schlesinger, M.I., Anochina, M.O.: Parsing and Recognition of Printed Notes. In: Proceedings of the conference Control Systems and Computers, pp. 30-38. Kiev (2003)

8. Schlesinger, M.I., Hlavac, V.: Ten Lectures on Statistical and Structural Pattern Recognition. Kluwer Academic Publishers, Dordrecht (2002)

9. Toyota, S., Uchida, S., Suzuki, M.: Structural Analysis of Mathematical Formulae with Verification Based on Formula Description Grammar. In: Procceedings of the 7th International Workshop DAS, pp. 153-163. Nelson, New Zealand (2006)

10. Toyozumi, K., Yamada, N., Mase, K., Kitasaka, T., Mori, K., Suenaga, Y., Takahashi, T.: A Study of Symbol Segmentation Method for Handwritten Mathematical Formula Recognition using Mathematical Structure Information. In: Proceedings of ICPR 2004, Cambridge, vol. 2, pp. 630-633 (2004)

11. http://www.nm.informatik.uni-muenchen.de/ensel/privat/pvmerlin 